\title{
P16 Assessment of Plaque Vulnerability Using a Novel Technique: Multi-spectral Photoacoustic Imaging (CVENT-PAI)
}

\author{
Yuki Imaizumi ${ }^{1{ }^{* *}}$, Hasan Obeid ${ }^{2}$, Pierre Julia ${ }^{2}$, David Calvet ${ }^{3}$, Pierre Boutouyrie ${ }^{2}$ \\ ${ }^{1}$ Université Paris Descartes, Paris, France \\ ${ }^{2}$ Université Paris Descartes and Hôpital Européen Georges Pompidou, Paris, France \\ ${ }^{3}$ Hôpital Sainte - Anne, Paris, France
}

\begin{abstract}
Background: Identification of intraplaque haemorrhage using magnetic resonance imaging (MRI) is expensive, technically demanding and sometimes uncomfortable. Photoacoustic imaging (PAI) is a new non-invasive technique combining multiwavelength infrared laser light and ultrasound (US) imaging, able to discriminate blood and other components in the tissues. The measurement by the multi-spectral PAI is likely to be more applicable and therefore could be complementary to MRI.

Objectives: Validate a portable multimodal and multi-wavelength PAI system, for the identification of intraplaque haemorrhage and compare with MRI.

Materials and Methods: Patients underwent carotid MRI, and US. Photoacoustic acquisitions are performed and analysed a posteriori blindly of the status of the subjects and results of MRI/US. All patients gave written informed consent. Histology of plaque will be performed as gold standard.

Results: We included 15 patients ( $71 \pm 5$ years old) with carotid stenosis grade of $>70 \%$ (NASCET). PAI imaging was feasible in all patients. On raw images, only clutter signal can be observed. After adequate post processing, carotid wall with the intima media and plaques were clearly visible with PAI. PAI signal from the lumen can be observed, also strong PAI signals are visible inside the plaque in a reproducible manner. Correspondence between MRI and PAI will be analysed later.

Conclusion: The use of the PAI technique for carotid plaque assessment is feasible, and PAI signal are observed inside the plaque. Further analyses are needed to assess the reflected PAI signal inside the plaque, for haemorrhage detection in the future.

(c) 2019 Association for Research into Arterial Structure and Physiology. Publishing services by Atlantis Press International B.V. This is an open access article distributed under the CC BY-NC 4.0 license (http://creativecommons.org/licenses/by-nc/4.0/).
\end{abstract}

